\section{Broschüre zur Babyhaut-Pflege}

Bei der Frage nach der optimalen Hautpflege von Säuglingen erhalten Eltern oft viele unterschiedliche Informationen. Aktuelle klinische Studien erlauben nun erste einheitliche und evidenzgestützte Empfehlungen. Eine Informationsbroschüre mit diesen Empfehlungen für Ärzte und Fachkreise ist kostenlos erhältlich und steht auch im

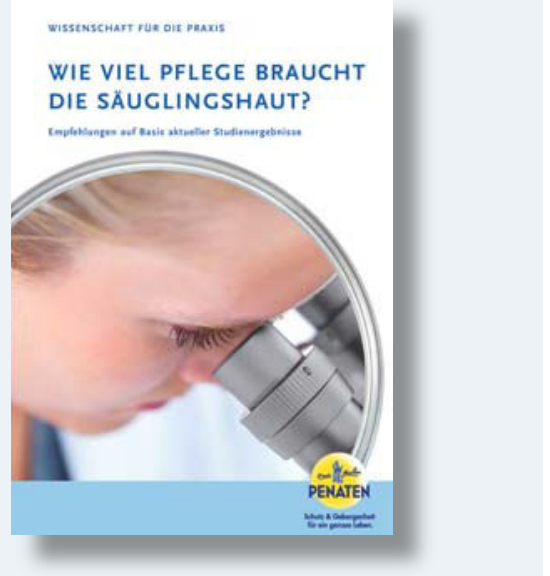

Internet zum Download zur Verfügung. Die Broschüre kann angefordert werden unter: Tel.: (o 69) 242861 65, Fax: (o 69) 24286250 oder E-Mail: annekathrin. raab@ketchumpleon.com.Sie kann außerdem unter www.penaten.de/hebammenforum heruntergeladen werden.

Nach Informationen von Johnson \& Johnson, Neuss

\section{Effektiv bei Kopflausbefall}

Eine aktuelle Studie bescheinigt auch dem neuen Dimeticon Präparat NYDA ${ }^{\circledR}$ sensitiv (ohne kosmetische Duft- und Zusatzstoffe) eine gute Wirksamkeit gegen Kopfläuse. Die geringfügig veränderte Konzentration des Wirkstoffs hat keinen Einfluss auf die Effektivität. Bei einer In-vitro-Untersuchung zeigten Läuse bereits fünf Minuten nach Applikation keine wesentlichen Lebenszeichen mehr. Der Studienleiter, Prof. Dr. Jörg Heukelbach aus Ceará, Brasilien, betonte: „Hochkonzentrierte DimeticonProdukte wie NYDA ${ }^{\circledR}$ und NYDA ${ }^{\circledR}$ sensitiv sind eine äußerst effektive und verträgliche Alternative zu den herkömmlichen Läusemitteln mit neurotoxischem Wirkprinzip."

Nach Informationen von Pohl-Boskamp Hohenlockstedt

\title{
Hämophilie: Innovativer Arzt-Patienten-Kontakt
}

- Anfang der 7oer-Jahre eingeführt, war die Heimselbstbehandlung für Hämophilie-Patienten ein großer Fortschritt. Nach individuellem Bedarf applizieren sich Betroffene die Medikation selbst und dokumentieren ihren Faktorverbrauch (gemäß Transfusionsgesetz) schriftlich in einem Tagebuch. Anhand der dokumentierten Daten kann der behandelnde Arzt dann seinen Patienten beim nächsten Termin beraten, die Therapie kontrollieren und gegebenenfalls umstellen. Nachteil der Methode: Die Arztbesuche liegen häufig weit auseinander, sodass auf Probleme erst spät reagiert werden kann. Auch der zeitliche Aufwand für die Patienten ist oft erheblich.

Mit dem elektronischen Substitutionstagebuch Haemoassist ${ }^{\circledR}$ soll die Therapietransparenz nun deutlich besser werden. Der Patient übermittelt mit einem Personal Digital Assistant (PDA) seine Daten an eine gesicherte Datenbank. Nur Arzt und Patient haben auf die mehrfach verschlüsselten Informationen Zugriff. Die Datenbank versendet bei Bedarf auch E-Mails an Therapeut und Betroffene-etwa wenn der errechnete Vorrat an Faktorkonzentrat zur Neige geht oder wenn bei kritischen Ereignissen wie ungewöhnlichen Blutungsmustern Handlungsbedarf entsteht.

„Das System kann früh entscheidende Hinweise geben, ob beispielsweise ein Patient von einer therapeutischen Faktorgabe besser auf eine Prophylaxe wechseln sollte“, erklärte Dr. Robert Klamroth, Berlin. Jeder Patient kann über den PDA seinen Arzt auch direkt kontaktieren, um im Notfall schnelle Hilfe anzufordern.

koc

Symposium „Das A und B der Hämophilie. Der Haemoassist ${ }^{\circledR}$ von Wyeth: Wegweiser der modernen Therapie“ und Meet-the-Expert „Öffnung des Haemoassist ${ }^{\circledR}$ von Wyeth“. Berlin, 26. September 2009. Veranstalter: Wyeth Pharma, Münster

\section{Bei Kindern und Jugendlichen auf zweite Masernimpfung achten}

_ Die WHO hatte sich zum Ziel gesetzt, das Masernvirus bis 2010 zu eliminieren. In vielen Ländern Europas - einschließlich Deutschland - ist man jedoch weit von diesem Ziel entfernt. Mit der Masernimpfung sollen vor allem die gefürchteten Komplikationen wie Enzephalitis und subakute sklerosierende Panenzephaltis (SPPE) verhindert werden. Je älter die erkrankten Personen, umso größer ist das Risiko für eine ZNS-Beteiligung, erklärte Prof. Dr. UIrich Heininger aus Basel. Denn bei einem Enzephalitis-Risiko von 1:500 bei jungen Erwachsenen könne man kaum noch von einer sehr seltenen Komplikation reden.

Von Ausnahmefällen abgesehen, wird die erste Masernimpfung mit zwölf Monaten empfohlen. Darauf sollte dann eine zweite Impfung (frühestens nach vier Wochen) folgen. Bei dieser Impfung handelt es sich nicht um eine Booster-Impfung im eigentlichen Sinne, erläuterte Heininger. Vielmehr möchte man Kindern, bei denen nach der ersten Impfung noch keine ausreichende Immunantwort erfolgte, ein „zweite Chance“ zur Antikörperbildung bieten. Die Daten unterstützen dieses Konzept: Nach der ersten Dosis beträgt die Serokonversionsrate etwa 96\%, nach der zweiten Dosis knapp 100\%. Heininger empfahl, bei allen Kindern, Jugendlichen und jungen Erwachsenen zu kontrollieren, ob die Masernimpfung mit zwei Impfdosen vollständig ist und ggf. nachzuimpfen. Nur so sei eine ausreichende Herdimmunität zu erreichen, durch die dann alle geschützt werden. Um Masern in einem Land vollständig zu eliminieren, müssen mehr als 95\% der Kinder zwei Impfdosen erhalten. Solche Raten wurden beispielsweise in Finnland erzielt, wo das Masernvirus heute als ausgerottet gilt. In Deutschland haben zweijährige Kinder zu etwa $86 \%$ zwei Impfdosen erhalten - von einer Elimination ist man also noch weit entfernt. $\quad$ mw

„Impfsymposium“ anlässlich des 3. Berliner Kongresses für Pädiatrie und der 20. Tagung der European Confederation of Primary Care Paediatricians. Berlin, 14. November 2009. Veranstalter: Sanofi Pasteur MSD, Leimen 